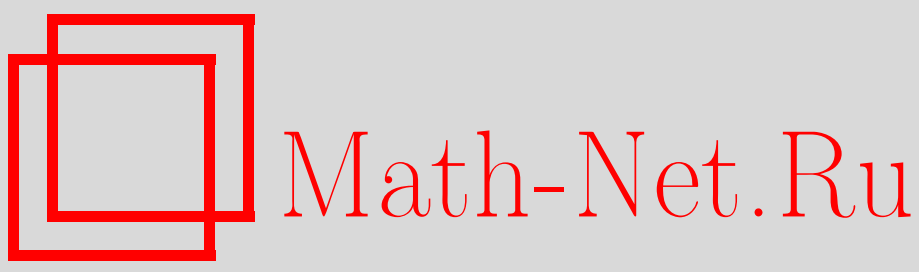

Н. Ю. Антонов, О сходимости почти всюду по кубам кратных тригонометрических рядов Фурье, Изв. РАН. Сер. матем., 2004, том 68, выпуск 2, 3-22

DOI: https://doi.org/10.4213/im472

Использование Общероссийского математического портала Math-Net.Ru подразумевает, что вы прочитали и согласны с пользовательским соглашением

http://www . mathnet.ru/rus/agreement

Параметры загрузки:

IP : 35.174 .16 .151

26 апреля 2023 г., 13:24:47 
УДК 517.518

\title{
Н.Ю. Антонов
}

\section{О сходимости почти всюду по кубам кратных тригонометрических рядов Фурье}

\begin{abstract}
Доказана теорема о том, что при некоторых условиях на функцию $\varphi:[0,+\infty) \rightarrow[0,+\infty)$ из сходимости почти всюду тригонометрических рядов Фурье всех функций из класса $\varphi(L)_{[-\pi, \pi)}$ следует сходимость по кубам кратного ряда Фурье и всех его сопряженных рядов любой функции $f \in \varphi(L)\left(\log ^{+} L\right)_{[-\pi, \pi)^{d}}^{d-1}, d \in \mathbb{N}$. Из этой теоремы и полученного автором ранее результата о сходимости почти всюду рядов Фурье функций одной переменной из класса $L\left(\log ^{+} L\right)\left(\log ^{+} \log ^{+} \log ^{+} L\right)_{[-\pi, \pi)}$ вытекает следующее утверждение: если $f \in L\left(\log ^{+} L\right)^{d}\left(\log ^{+} \log ^{+} \log ^{+} L\right)_{[-\pi, \pi)^{d}}, d \in \mathbb{N}$, то ряд Фурье функции $f$ и все его сопряженные ряды сходятся по кубам почти всюду.

Библиография: 15 наименований.
\end{abstract}

\section{§1. Введение}

Пусть $d$ - натуральное число, $\mathbb{T}^{d}=[-\pi, \pi)^{d}-d$-мерньй тор, $\mathbb{Z}^{d}$ - целочисленная решетка в $\mathbb{R}^{d}, k=\left(k_{1}, \ldots, k_{d}\right) \in \mathbb{Z}^{d}, x=\left(x_{1}, \ldots, x_{d}\right) \in \mathbb{T}^{d}, k x=k_{1} x_{1}+\cdots$ $\cdots+k_{d} x_{d}$

$$
\sum_{k \in \mathbb{Z}^{d}} a_{k} e^{i k x}
$$

- кратный тригонометрический ряд Фурье суммируемой на $\mathbb{T}^{d}$ функции $f$, a

$$
S_{n}(f, x)=S_{n}^{d}(f, x)=\sum_{k=\left(k_{1}, \ldots, k_{d}\right): \max \left|k_{i}\right| \leqslant n} a_{k} e^{i k x}
$$

- его $n$-я кубическая частичная сумма.

Пусть $B$ - некоторое непустое подмножество множества первых $d$ натуральных чисел: $B=\left\{r_{1}, \ldots, r_{l}\right\} \subset\{1, \ldots, d\}$. Ряд

$$
\sum_{k \in \mathbb{Z}^{d}} \prod_{j=1}^{l}\left(-i \operatorname{sign} k_{r_{j}}\right) a_{k} e^{i k x}
$$

называется сопряженным $к$ ряду (1.1) по переменным, номера которых входят во множество $B$, или $B$-сопряженным, а $n$-я кубическая частичная сумма

Работа выполнена при финансовой поддержке Российского фонда фундаментальных исследований (гранты № 02-01-00764 и 02-01-06042) и Программы поддержки ведущих научных школ (грант № 00-15-96035). 
$\widetilde{S}_{n, B}(f, x)=\widetilde{S}_{n, B}^{d}(f, x)$ ряда $(1.2)$ определяется аналогично $n$-й кубической частичной сумме ряда (1.1).

Всего для ряда (1.1) можно определить $2^{d}-1$ сопряженных рядов. Заметим, что, взяв в качестве $B$ пустое множество, можно полагать ряд (1.1) $B$-сопряженным ( $\varnothing$-сопряженным $)$ к самому себе. Отметим также, что введенные здесь для $d \geqslant 1$ определения при $d=1$ совпадают с обычными определениями тригонометрического ряда и его сопряженного ряда для $2 \pi$-периодической функции.

Пусть $\varphi:[0,+\infty) \rightarrow[0,+\infty)$ - неубывающая функция. Обозначим через $\varphi(L)=$ $\varphi(L)\left(\mathbb{T}^{d}\right)$ множество всех определенных на $\mathbb{T}^{d}$ и измеримых по Лебегу функций $f$, удовлетворяюших условию

$$
\int_{\mathbb{T}^{d}} \varphi(|f(t)|) d t<\infty
$$

Заметим, что если $\varphi_{1}(u)=O\left(\varphi_{2}(u)\right)$ при $u \rightarrow \infty$, то $\varphi_{2}(L) \subset \varphi_{1}(L)$.

В случае $d=1$ Л. Карлесон [1] доказал, что если $f \in L^{2}(\mathbb{T})$, то тригонометрический ряд Фурье функции $f$ сходится почти всюду. Затем Р. Хант [2] обобшил утверждение о сходимости почти всюду рядов Фурье на функщии из классов $L^{p}(\mathbb{T}), \quad p>1$, и $L\left(\log ^{+} L\right)^{2}(\mathbb{T})$, а П. Шёлин [3] - на функции из класса $L\left(\log ^{+} L\right)\left(\log ^{+} \log ^{+} L\right)(\mathbb{T})$. Автором [4] было установлено, что условие $f \in L\left(\log ^{+} L\right)\left(\log ^{+} \log ^{+} \log ^{+} L\right)(\mathbb{T})$ также является достаточным для сходимости почти всюду ряда Фурье функции $f$. С другой стороны, С.В. Конягиным [5] доказано, что для любой неубывающей функции $\varphi:[0,+\infty) \rightarrow[0,+\infty)$, удовлетворяющей условию $\varphi(u)=o(u \sqrt{\ln u} / \sqrt{\ln \ln u})$ при $u \rightarrow \infty$, найдется функция из класса $\varphi(L)$ такая, что ее ряд Фурье неограниченно расходится всюду на $\mathbb{T}$.

Пусть $d \geqslant 2$. Ряд (1.1) (соответственно, (1.2)) сходится по кубам (в случае $d=2$ - по квадратам) в точке $x_{0} \in \mathbb{T}^{d}$, если последовательность $S_{n}\left(f, x_{0}\right)$ (соответственно, $\left.\widetilde{S}_{n, B}\left(f, x_{0}\right)\right)$ имеет предел при $n \rightarrow \infty$.

Сходимость почти всюду по квадратам рядов Фурье функций $f \in L^{2}\left(\mathbb{T}^{2}\right)$ была установлена Н.Р. Тевзадзе [6]. Ч. Фефферман [7] распространил этот результат на функции $f \in L^{p}\left(\mathbb{T}^{d}\right), p>1, d \geqslant 2$, а затем П.Шёлин [8] доказал, что если $f \in L\left(\log ^{+} L\right)^{d}\left(\log ^{+} \log ^{+} L\right)\left(\mathbb{T}^{d}\right)$, то ее ряд Фурье сходится по кубам почти всюду. Для доказательства последнего результата Шёлин использовал свою теорему [3] о сходимости п. в. рядов Фурье функций из $L\left(\log ^{+} L\right)\left(\log ^{+} \log ^{+} L\right)$ в одномерном случае.

Наилучший в настояшее время результат, касающийся расходимости по кубам на множестве положительной меры кратных рядов Фурье функций из $\varphi(L)\left(\mathbb{T}^{d}\right)$, $d \geqslant 2$, также принадлежит С.В. Конягину [9]: для любой функции $\varphi(u)=$ $o\left(u(\log u)^{d-1} \log \log u\right)$ при $u \rightarrow \infty$ существует $f(x) \in \varphi(L)\left(\mathbb{T}^{d}\right)$ с расходящимся всюду по кубам рядом Фурье.

В настоящей работе доказана теорема, позволяюшая переносить результаты о сходимости почти всюду обычных рядов Фурье функций из классов $\varphi(L)$ на кратные ряды Фурье в случае сходимости по кубам.

Неотрицательную неубывающую функцию $\psi(u)$ назовем медленно растущей, если при любом $\delta>0$ функция $\psi(u) u^{-\delta}$ убывает при достаточно больших $u$. 
Будем говорить, что функция $\varphi:[0,+\infty) \rightarrow[0,+\infty)$ удовлетворяет условия.м (1.3) если

$$
\begin{array}{cl}
\varphi(0)=0, & \varphi(u)-\text { выпуклая и возрастающая на }[0,+\infty), \\
& \varphi\left(u^{\frac{1}{2}}\right)-\text { вогнутая на }[0,+\infty) .
\end{array}
$$

Далее будем полагать $\log u=\log _{2} u$.

ТЕОРема 1. Пусть $\psi(u)$-медленно растущая функиия, и пусть для любого натурального д существует константа и такая, что функиия

$$
\varphi_{d}(u)=u \psi\left(u+u_{d}\right)\left(\log \left(u+u_{d}\right)\right)^{d-1}
$$

удовлетворяет условиям (1.3). Предположим, что для любой функиии $f \in$ $\varphi_{1}(L)(\mathbb{T})$

$$
\lim _{n \rightarrow \infty} S_{n}(f, x)=f(x) \quad \text { n. } \boldsymbol{\theta} .
$$

Тогда для любого $d \in \mathbb{N}$, если $f \in \varphi_{d}(L)\left(\mathbb{T}^{d}\right)$, ряд Фурье функции $f$ и все его сопряженные ряды сходятся по кубам почти всюду.

Следующее утверждение есть следствие теоремы 1 и результата автора о сходимости почти всюду одномерных рядов Фурье функций из класса

$$
L\left(\log ^{+} L\right)\left(\log ^{+} \log ^{+} \log ^{+} L\right)(\mathbb{T})
$$

Teоpema 2. Пусть $d \in \mathbb{N}, f \in L\left(\log ^{+} L\right)^{d}\left(\log ^{+} \log ^{+} \log ^{+} L\right)\left(\mathbb{T}^{d}\right)$. Тогда ряд Фурье функиии $f$ и все его сопряженные ряды сходятся по кубам почти всюду.

\section{§2. Вспомогательные утверждения}

Сформулируем известные результаты и получим вспомогательные утверждения, которые понадобятся нам при доказательстве теоремы 1.

Говорят, что функция $\widetilde{\varphi}:[0,+\infty) \rightarrow[0,+\infty)$ удовлетворяет $\Delta_{2}$-условию, если $\widetilde{\varphi}(2 u=O(\widetilde{\varphi}(u))$ при $u \rightarrow \infty$.

Лемма 1. Пусть неубывающая непрерывная функиия $\widetilde{\varphi}:[0,+\infty) \rightarrow[0,+\infty)$ удовлетворяет $\Delta_{2}$-условию и $\widetilde{\varphi}(0)=0$. Тогда для любой функции $h \in \widetilde{\varphi}(L)\left(\mathbb{T}^{d}\right)$ и любого числа $\varepsilon>0$ найдется кратный тригонометрический полином $g(t)$ такой, что

$$
\int_{\mathbb{T}^{d}} \widetilde{\varphi}(|h(t)-g(t)|) d t<\varepsilon
$$

Отметим, что в случае $d=1$ утверждение, более общее, чем лемма 1 , получено П. Л. Ульяновым [10, следствие 3.2]. 
ДокАЗАТЕЛЬСТво. Поскольку функция $\widetilde{\varphi}$ непрерьвна и $\widetilde{\varphi}(0)=0$, найдется число $u_{0}>0$ такое, что $0<\widetilde{\varphi}\left(u_{0}\right)<\varepsilon /\left(2(2 \pi)^{d}\right)$. Так как $\widetilde{\varphi}$ удовлетворяет $\Delta_{2}$-условию, найдется $C>0$ такое, что для всех $u \geqslant u_{0}$

$$
\widetilde{\varphi}(2 u) \leqslant C \widetilde{\varphi}(u) .
$$

Пусть $x \geqslant u_{0}, \alpha \geqslant 1$. Выберем натуральное $n$ таким, что $2^{n-1} \leqslant \alpha<2^{n}$. Тогда

$$
\widetilde{\varphi}(\alpha x) \leqslant \widetilde{\varphi}\left(2^{n} x\right) \leqslant C^{n} \widetilde{\varphi}(x)=C\left(2^{n-1}\right)^{\log C} \leqslant C \alpha^{\log C} \widetilde{\varphi}(x),
$$

T. e.

$$
\widetilde{\varphi}(\alpha x) \leqslant C \alpha^{p} \widetilde{\varphi}(x), \quad x \geqslant u_{0}, \quad p=\log C .
$$

Поскольку множество тригонометрических полиномов ( $d$ переменных) всюду плотно в пространстве $L^{p}\left(\mathbb{T}^{d}\right)$, найдется полином $g(t)$, удовлетворяюший условию

$$
\int_{\mathbb{T}^{d}}|h(t)-g(t)|^{p} d t<\frac{\varepsilon u_{0}^{p}}{2 C \widetilde{\varphi}\left(u_{0}\right)} .
$$

Покажем, что выбранный полином $g$ удовлетворяет условию (2.1). Ясно, что

$$
\begin{aligned}
\int_{\mathbb{T}^{d}} \tilde{\varphi}(|h(t)-g(t)|) d t= & \int_{\left\{t \in \mathbb{T}^{d}:|h(t)-g(t)| \leqslant u_{0}\right\}} \widetilde{\varphi}(|h(t)-g(t)|) d t \\
& +\int_{\left\{t \in \mathbb{T}^{d}:|h(t)-g(t)|>u_{0}\right\}} \widetilde{\varphi}(|h(t)-g(t)|) d t .
\end{aligned}
$$

В силу выбора $u_{0}$ и неубывания $\widetilde{\varphi}$ первое слагаемое в правой части $(2.4)$ меньше $\varepsilon / 2$. Для второго слагаемого, применяя $(2.2)$ при $\alpha=|h(t)-g(t)| / u_{0}$ и $x=u_{0}$, а затем (2.3), получаем

$$
\begin{aligned}
& \int_{\left\{t \in \mathbb{T}^{d}:|h(t)-g(t)|>u_{0}\right\}} \widetilde{\varphi}(|h(t)-g(t)|) d t \\
& \quad \leqslant \frac{C \widetilde{\varphi}\left(u_{0}\right)}{u_{0}^{p}} \int_{\left\{t \in \mathbb{T}^{d}:|h(t)-g(t)|>u_{0}\right\}}|h(t)-g(t)|^{p} d t<\frac{\varepsilon}{2} .
\end{aligned}
$$

Лемма доказана.

Следуюшее утверждение является непосредственным следствием леммы 1.

Лемма 2. Пусть функиия $\varphi:[0,+\infty) \rightarrow[0,+\infty)$ удовлетворяет условиям (1.3). Тогда для любых чисел $\varepsilon, a>0$ и любой функции $h \in \varphi(L)\left(\mathbb{T}^{d}\right)$ найдется кратный тригонометрический полином $g(t)$ такой, что

$$
\begin{array}{r}
\int_{\mathbb{T}^{d}}|h(t)-g(t)| d t<\varepsilon, \\
\int_{\mathbb{T}^{d}} \varphi(a|h(t)-g(t)|) d t<\varepsilon .
\end{array}
$$


ДоКАЗАТЕЛЬСТВО. Из того, что $\varphi\left(u^{1 / 2}\right)$ вогнутая и $\varphi(0)=0$, следует, что $\varphi(u)$ удовлетворяет $\Delta_{2}$-условию. Положим $\widetilde{\varphi}(u)=u+\varphi(a u)$. Ясно, что функция $\widetilde{\varphi}$ также удовлетворяет $\Delta_{2}$-условию. Применяя к $\widetilde{\varphi}$ лемму 1 , из неравенства $(2.1)$ получаем (2.5) и (2.6). Лемма доказана.

Напомним, что оператор $V$ является оператором типа $(\varphi, \varphi)$, если сушествует константа $A>0$ такая, что для всех $f \in \varphi(L)$

$$
\int_{\mathbb{T}^{d}} \varphi(|V(f, x)|) d x \leqslant \int_{\mathbb{T}^{d}} \varphi(A|f(x)|) d x .
$$

Оператор $V$ инвариантен относительно сдвига, если

$$
V(f(\cdot+s), x)=V(f(\cdot), x+s), \quad x, s \in \mathbb{T}^{d} .
$$

Teоpema A [11, с. 154, теорема 3]. Пусть $V_{n}$ - последовательность линейных операторов, каждый из которых является оператором типа $(\varphi, \varphi)$ u инвариантен относительно сдвига. Пусть функиия $\varphi$ удовлетворяет условиям (1.3). Предположим, что для каждой функиии $f$ из $\varphi(L)$

$$
\limsup _{n \rightarrow \infty}\left|V_{n}(f, x)\right|<\infty
$$

для всех $x$ из некоторого (зависящего от $f$ ) множества положительной меры. Пусть $V^{*}(f, x)=\sup _{n \geqslant 1}\left|V_{n}(f, x)\right|$. Тогда найдется положительное число $A$ такое, ито для всех $f \in \varphi(L)$

$$
m\left\{x: V^{*}(f, x)>\alpha\right\} \leqslant \int_{\mathbb{T}^{d}} \varphi\left(\frac{A}{\alpha}|f(x)|\right) d x, \quad \alpha>0 .
$$

Пусть $d \in \mathbb{N}, B=\left\{r_{1}, \ldots, r_{k}\right\}$ - непустое подмножество множества $\{1,2, \ldots, d\}$, $|B|=k ; B$-сопряженному ряду функции $f \in L\left(\mathbb{T}^{d}\right)$ сопоставим $B$-сопряженную функцию $\tilde{f}_{B}(x)$, определяемую следуюшим образом. Пусть $\varepsilon(B)=\left(\varepsilon_{r_{1}}, \ldots, \varepsilon_{r_{k}}\right)$,

$$
T_{\varepsilon(B)}^{|B|}=\prod_{j=1}^{k}\left(\mathbb{T} \backslash\left[-\varepsilon_{r_{j}}, \varepsilon_{r_{j}}\right]\right),
$$

$d$-мерньй вектор $t(B)$ имеет координаты

$$
t_{\nu}(B)= \begin{cases}t_{\nu}, & \text { если } \nu=r_{j}, j=1, \ldots, k \\ 0 & \text { при остальных } \nu .\end{cases}
$$

Положим

$$
\tilde{f}_{B}(x)=\left(-\frac{1}{2 \pi}\right)^{k} \lim _{\varepsilon(B) \rightarrow+0} \int_{T_{\varepsilon(B)}^{|B|}} f(x+t(B))\left(\prod_{j=1}^{k} \operatorname{ctg}\left(\frac{t_{r_{j}}}{2}\right)\right) d t_{r_{1}} \ldots d t_{r_{k}} .
$$

Предел в (2.7) берется при независимом стремлении к +0 компонент вектора $\varepsilon(B)$. В случае, когда $B$ - пустое множество, будем по определению полагать, что $B$-сопряженная функция совпадает с $f(x): \tilde{f}_{B}(x)=\tilde{f}_{\varnothing}(x)=f(x)$. 
Пусть $D_{n}(t)$ и $\widetilde{D}_{n}(t)$ - соответственно ядро Дирихле и сопряженное с ним ядро:

$$
D_{n}(t)=\frac{\sin \left(n+\frac{1}{2}\right) t}{2 \sin \left(\frac{t}{2}\right)}, \quad \widetilde{D}_{n}(t)=\frac{\cos \left(\frac{t}{2}\right)-\cos \left(n+\frac{1}{2}\right) t}{2 \sin \left(\frac{t}{2}\right)} .
$$

Тогда $n$-ю кубическую сумму ряда (1.2) можно представить следующим образом:

$$
\widetilde{S}_{n, B}(f, x)=\frac{1}{\pi^{d}} \int_{\mathbb{T}^{d}} \prod_{i \in B}\left(-\widetilde{D}\left(t_{i}\right)\right) \prod_{j \in\{1, \ldots, d\} \backslash B} D_{n}\left(t_{j}\right) f(x+t) d t,
$$

где $B$ может быть и пустым, а произведение $\prod$, в котором множество сомножителей пусто, по определению считаем равным единице.

Лемма 3. Пусть $d \in \mathbb{N}, \quad B \subset\{1,2, \ldots, d\}$. Предположим, ито удовлетворяющая условиям (1.3) функиия $\varphi$ такова, что $u(\log u)^{d-1}=O(\varphi(u))$ при $u \rightarrow \infty$. Тогда эквивалентны следующие три условия:

1) если $f \in \varphi(L)\left(\mathbb{T}^{d}\right)$, то для почти всех $x \in \mathbb{T}^{d}$

$$
\lim _{n \rightarrow \infty} \widetilde{S}_{n, B}(f, x)=\tilde{f}_{B}(x)
$$

2) найдется константа $A_{1}>0$ такая, что для всех $f \in \varphi(L)\left(\mathbb{T}^{d}\right)$ u y $>0$

$$
m\left\{x \in \mathbb{T}^{d}: \sup _{n \geqslant 1}\left|\widetilde{S}_{n, B}(f, x)\right|>y\right\} \leqslant \int_{\mathbb{T}^{d}} \varphi\left(\frac{A_{1}}{y}|f(t)|\right) d t
$$

3) найдутся константы $A>0$ и $y_{0} \geqslant 0$ такие, что для любого кратного тригонометрического полинома $g$, определенного на $\mathbb{T}^{d}$, и любого числа y $>y_{0}$ справедливо неравенство

$$
m\left\{x \in \mathbb{T}^{d}: \max _{n \geqslant 1}\left|\widetilde{S}_{n, B}(g, x)\right|>y\right\} \leqslant \frac{A}{y}\left(\int_{\mathbb{T}^{d}} \varphi(|g(t)|) d t+1\right) .
$$

ДокАЗАТЕЛЬСТво. Как нетрудно видеть, условие 2) следует из 1) в силу теоремы А. Докажем, что условие 2 ) влечет 1 ).

Пусть $f \in \varphi(L)\left(\mathbb{T}^{d}\right)$. Для любого $y>0$

$$
m\left\{x \in \mathbb{T}^{d}:\left|\tilde{f}_{B}(x)\right|>y\right\} \leqslant \frac{C}{y}\left(\int_{\mathbb{T}^{d}}|f(t)|\left(\log ^{+}|f(t)|\right)^{d-1} d t+1\right) .
$$

Если $B$ непусто, то при $d=1$ это есть (с точностью до +1 в правой части) неравенство Колмогорова [12] для сопряженных функций, при $d \geqslant 2$ неравенство (2.10) является частным случаем результата Л.В. Жижиашвили [13]. Если $B=\{\varnothing\}$, то (2.10) следует из того, что для любых $y>0$

$$
y \cdot m\left\{t \in \mathbb{T}^{d}:|f(t)|>y\right\} \leqslant \int_{\left\{t \in \mathbb{T}^{d}:|f(t)|>y\right\}}|f(t)| d t \leqslant \int_{\mathbb{T}}|f(t)| d t .
$$


Согласно условию леммы для некоторых $C_{1}>0$ и $u_{0}>2$ при $u \geqslant u_{0}$ справедливо неравенство $u(\log u)^{d-1} \leqslant C_{1} \varphi(u)$, поэтому

$$
\begin{aligned}
& \int_{\mathbb{T}^{d}}|f(t)|\left(\log ^{+}|f(t)|\right)^{d-1} d t \\
& \quad=\left(\int_{\left\{t \in \mathbb{T}^{d}:|f(t)| \leqslant u_{0}\right\}}+\int_{\left\{t \in \mathbb{T}^{d}:|f(t)|>u_{0}\right\}}\right)|f(t)|\left(\log ^{+}|f(t)|\right)^{d-1} d t \\
& \quad \leqslant(2 \pi)^{d} u_{0}\left(\log u_{0}\right)^{d-1}+C_{1} \int_{\mathbb{T}^{d}} \varphi(|f(t)|) d t .
\end{aligned}
$$

Отсюда и из неравенства (2.10) получаем

$$
m\left\{x \in \mathbb{T}^{d}:\left|\tilde{f}_{B}(x)\right|>y\right\} \leqslant \frac{C_{2}}{y}\left(\int_{\mathbb{T}^{d}} \varphi(|f(t)|) d t+1\right),
$$

где $C_{2}=\max \left\{C C_{1}, C(2 \pi)^{d} u_{0}\left(\log u_{0}\right)^{d-1}\right\}$.

В силу выпуклости функции $\varphi(u)$ и условия $\varphi(0)=0$ справедливо неравенство

$$
\varphi\left(\frac{A_{1}|f(t)|}{y}\right) \leqslant \frac{A_{1}}{y} \varphi(|f(t)|), \quad 0 \leqslant \frac{A_{1}}{y} \leqslant 1, \quad t \in \mathbb{T}^{d} .
$$

Отсюда и из (2.9) следует

$$
m\left\{x \in \mathbb{T}^{d}: \sup _{n \geqslant 1}\left|\widetilde{S}_{n, B}(f, x)\right|>y\right\} \leqslant \frac{A_{1}}{y} \int_{\mathbb{T}^{d}} \varphi(|f(t)|) d t
$$

при $y>A_{1}$.

Обозначим $U_{n, B}(f, x)=\widetilde{S}_{n, B}(f, x)-\tilde{f}_{B}(x)$. Поскольку

$$
\begin{aligned}
\{x & \left.\in \mathbb{T}^{d}: \sup _{n \geqslant 1}\left|U_{n, B}(f, x)\right|>y\right\} \\
& \subset\left\{x \in \mathbb{T}^{d}: \sup _{n \geqslant 1}\left|\widetilde{S}_{n, B}(f, x)\right|>\frac{y}{2}\right\} \cup\left\{x \in \mathbb{T}^{d}: \sup _{n \geqslant 1}\left|\tilde{f}_{B}(x)\right|>\frac{y}{2}\right\},
\end{aligned}
$$

то из неравенств $(2.13)$ и (2.11) следует

$$
m\left\{x \in \mathbb{T}^{d}: \sup _{n \geqslant 1}\left|U_{n, B}(f, x)\right|>y\right\} \leqslant \frac{K}{y}\left(\int_{\mathbb{T}^{d}} \varphi(|f(t)|) d t+1\right), \quad y>A_{1},
$$

где $K=2\left(C_{2}+A_{1}\right)$.

Далее зафиксируем произвольные числа $\theta>0$ и $\alpha>A_{1}$. Согласно лемме 2 , примененной при $\varepsilon=1, a=1, h=\theta f$, найдется тригонометрический полином $g(x)=g_{\theta, f}(x)$ такой, что

$$
\int_{\mathbb{T}^{d}} \varphi(|\theta f(t)-g(t)|) d t<1
$$


Частичные суммы $B$-сопряженного ряда тригонометрического полинома, начиная с некоторого номера $n$, совпадают с $B$-сопряженной функцией этого полинома. Поэтому

$$
\lim _{n \rightarrow \infty} U_{n, B}(g, x)=0 .
$$

Используя это равенство, имеем

$$
\begin{aligned}
m\left\{x \in \mathbb{T}^{d}: \limsup _{n \rightarrow \infty}\left|U_{n, B}(f, x)\right|>\frac{\alpha}{\theta}\right\} \\
=m\left\{x \in \mathbb{T}^{d}: \limsup _{n \rightarrow \infty}\left|U_{n, B}(\theta f, x)\right|>\alpha\right\} \\
=m\left\{x \in \mathbb{T}^{d}: \limsup _{n \rightarrow \infty}\left|U_{n, B}(\theta f, x)-U_{n, B}(g, x)\right|>\alpha\right\} \\
=m\left\{x \in \mathbb{T}^{d}: \limsup _{n \rightarrow \infty}\left|U_{n, B}(\theta f-g, x)\right|>\alpha\right\} .
\end{aligned}
$$

Отсюда, применяя неравенство (2.14) к функции $\theta f(t)-g(t)$, а затем (2.15), получаем

$$
\begin{aligned}
& m\left\{x \in \mathbb{T}^{d}: \limsup _{n \rightarrow \infty}\left|U_{n, B}(f, x)\right|>\frac{\alpha}{\theta}\right\} \\
& \quad \leqslant \frac{K}{\alpha}\left(\int_{\mathbb{T}^{d}} \varphi(|\theta f(t)-g(t)|) d t+1\right)<\frac{2 K}{\alpha} .
\end{aligned}
$$

Поскольку $\theta>0$ и $\alpha>A_{1}$ выбраны произвольно, можно взять $\theta=\alpha / y(y>0)$. Тогда, переходя в $(2.16)$ к пределу при $\alpha \rightarrow \infty$, для любого $y>0$ получим

$$
m\left\{x \in \mathbb{T}^{d}: \limsup _{n \rightarrow \infty}\left|U_{n, B}(f, x)\right|>y\right\}=0 .
$$

Теперь, пользуясь тем, что

$$
\begin{aligned}
\{x & \left.\in \mathbb{T}^{d}: \limsup _{n \rightarrow \infty}\left|U_{n, B}(f, x)\right|>0\right\} \\
& =\bigcup_{k=1}^{\infty}\left\{x \in \mathbb{T}^{d}: \limsup _{n \rightarrow \infty}\left|U_{n, B}(f, x)\right|>\frac{1}{k}\right\},
\end{aligned}
$$

и, следовательно,

$$
\begin{aligned}
m\left\{x \in \mathbb{T}^{d}: \limsup _{n \rightarrow \infty}\left|U_{n, B}(f, x)\right|>0\right\} \\
\quad \leqslant \sum_{k=1}^{\infty} m\left\{x \in \mathbb{T}^{d}: \limsup _{n \rightarrow \infty}\left|U_{n, B}(f, x)\right|>\frac{1}{k}\right\}
\end{aligned}
$$

и, замечая, что в силу (2.17) все члены ряда в правой части последнего неравенства равны нулю, получаем

$$
m\left\{x \in \mathbb{T}^{d}: \limsup _{n \rightarrow \infty}\left|U_{n, B}(f, x)\right|>0\right\}=0,
$$


т. е. для почти всех $x \in \mathbb{T}^{d}$

$$
\lim _{n \rightarrow \infty} \widetilde{S}_{n, B}(f, x)=\tilde{f}_{B}(x)
$$

Таким образом, мы установили эквивалентность условий 1) и 2). Нам осталось доказать эквивалентность условий 2) и 3 ). Предположим, что выполнено условие 2). Замечая, что произвольный полином есть функция ограниченная, а значит, принадлежит $\varphi(L)\left(\mathbb{T}^{d}\right)$, и применяя ранее полученное неравенство $(2.12)$, мы видим, что условие 3 ) выполняется с константами $A=A_{1}$ и $y_{0}=A_{1}$.

Наконец, докажем, что из условия 3 ) следует 2 ).

Пусть $f \in \varphi(L)\left(\mathbb{T}^{d}\right), B \subset\{1,2, \ldots, d\}$. Зафиксируем $y>y_{0}+1$ и $n \in \mathbb{N}$. Обозначим

$$
M_{n, B}(f, x)=\max _{1 \leqslant k \leqslant n}\left|\widetilde{S}_{k, B}(f, x)\right|, \quad M_{B}(f, x)=\sup _{n \in \mathbb{N}}\left|\widetilde{S}_{n, B}(f, x)\right| .
$$

Согласно лемме 2 найдется тригонометрический полином $g(t)$ такой, что выполняются условия (2.5) и (2.6) при $\varepsilon=1 /(n+1)^{d}, a=2, h=f$. Тогда с помошью представления (2.8) и неравенства (2.5) имеем для $x \in \mathbb{T}^{d}$ и $k \in\{1,2, \ldots, n\}$

$$
\left|\widetilde{S}_{k, B}(f, x)-\widetilde{S}_{k, B}(g, x)\right|=\left|\widetilde{S}_{k, B}(f-g, x)\right| \leqslant(n+1)^{d} \int_{\mathbb{T}^{d}}|f(t)-g(t)| d t<1,
$$

откуда

$$
M_{n, B}(f, x) \leqslant M_{n, B}(g, x)+1, \quad x \in \mathbb{T}^{d} .
$$

Используя это неравенство, получаем вложение

$$
\left\{x \in \mathbb{T}^{d}: M_{n, B}(f, x)>y\right\} \subset\left\{x \in \mathbb{T}^{d}: M_{n, B}(g, x)>y-1\right\} .
$$

Из выпуклости функции $\varphi$ и неравенства (2.6) следует

$$
\begin{aligned}
\int_{\mathbb{T}^{d}} \varphi & (|g(t)|) d t \leqslant \frac{1}{2} \int_{\mathbb{T}^{d}} \varphi(2|f(t)-g(t)|) d t \\
& +\frac{1}{2} \int_{\mathbb{T}^{d}} \varphi(2|f(t)|) d t<\frac{1}{2} \int_{\mathbb{T}^{d}} \varphi(2|f(t)|) d t+\frac{1}{2(n+1)^{d}} .
\end{aligned}
$$

Применяя последовательно (2.19), условие 3) леммы и (2.20), имеем

$$
\begin{aligned}
m\{x & \left.\in \mathbb{T}^{d}: M_{n, B}(f, x)>y\right\} \leqslant m\left\{x \in \mathbb{T}^{d}: M_{n, B}(g, x)>y-1\right\} \\
& \leqslant \frac{A}{y-1}\left(\int_{\mathbb{T}^{d}} \varphi(|g(t)|) d t+1\right)<\frac{A}{y-1}\left(\frac{1}{2} \int_{\mathbb{T}^{d}} \varphi(2|f(t)|) d t+\frac{1}{2(n+1)^{d}}+1\right) \\
& <\frac{2 A}{y-1}\left(\int_{\mathbb{T}^{d}} \varphi(2|f(t)|) d t+1\right) .
\end{aligned}
$$

Отсюда, учитывая, что $M_{B}(f, x)=\lim _{n \rightarrow \infty} M_{n, B}(f, x)$, получаем

$$
m\left\{x \in \mathbb{T}^{d}: M_{B}(f, x)>y\right\}<\frac{2 A}{y-1}\left(\int_{\mathbb{T}^{d}} \varphi(2|f(t)|) d t+1\right) .
$$


Выбрав у достаточно большим, можно сделать правую часть последнего неравенства сколь угодно малой, в частности, меньшей, чем $m \mathbb{T}^{d}=(2 \pi)^{d}$. Следовательно, для произвольной функции $f \in \varphi(L)\left(\mathbb{T}^{d}\right)$ соотношение $M_{B}(f, x)<+\infty$ выполнено для всех $x$ из некоторого множества положительной меры. Применяя теорему А, получаем условие 2). Лемма доказана.

Положительная функция $b(u)$, определенная для $u \geqslant u_{0} \geqslant 0$, назьвается медленно колеблющейся, если при любом $\delta>0$ функция $b(u) u^{\delta}$ при достаточно больших $u$ возрастает, а $b(u) u^{-\delta}$ убывает. Заметим, что согласно данному нами во введении определению медленно растущая функция есть частньй случай медленно колеблющейся.

Tеорема В [14, с. 179, теорема 4.39]. Предположим, что $\chi(u)=u \psi(u)$, где $\psi(u)$ - медленно колеблющаяся функиия, положсительная, за исключением окрестности точки $u=0$, где она равна нулю. Тогда для функиии $\varphi$, определенной равенством

$$
\varphi(u)=u \int_{0}^{u} t^{-2} \chi(t) d t
$$

имеем

$$
\int_{\mathbb{T}} \chi(|\tilde{f}(t)|) d t \leqslant K \int_{\mathbb{T}} \varphi(|f(t)|) d t+K
$$

для любой $f \in \varphi(L)(\mathbb{T})$, әде $K=K(\psi)$ не зависит от $f$.

Лемма 4. Пусть $\psi(u)$ - медленно растущая функиия, и пусть для $d \in \mathbb{N}$ функции $\varphi_{d}(u)$ определяются следующим образом:

$$
\varphi_{d}(u)=u \psi(u)\left(\log \left(u+u_{d}\right)\right)^{d-1}
$$

где $u_{d} \geqslant 1, u_{d} \geqslant u_{d-1}, \quad d \geqslant 2,-$ константы. Тогда для кажсдого $d \geqslant 2$ неравенство

$$
\int_{\mathbb{T}} \varphi_{d-1}(|\tilde{f}(t)|) d t \leqslant K_{d} \int_{\mathbb{T}} \varphi_{d}(|f(t)|) d t+K_{d}
$$

где $K_{d}=K_{d}(\psi)$ не зависит от $f$, справедливо для всех $f \in \varphi_{d}(L)(\mathbb{T})$.

ДокАЗАТЕльство. Нетрудно видеть, что произведение двух медленно растущих функций также является медленно растущей функцией. Поэтому из того, что $\psi(u)$ - медленно растущая, следует, что все функции $\varphi_{d} / u, d \geqslant 1$, также медленно растушие.

Пусть $d \geqslant 2$. Поскольку $\varphi_{d-1}(u) / u$ - медленно растушая функция, то функция $\varphi_{d-1}^{0}(u)$, совпадающая с $\varphi_{d-1}(u)$ при $u>1$ и равная нулю, если $0 \leqslant u \leqslant 1$, 
удовлетворяет условиям теоремы В. Применяя теорему В при $\chi(u)=\varphi_{d-1}^{0}(u)$, имеем

$$
\begin{aligned}
\int_{\mathbb{T}} \varphi_{d-1}(|\tilde{f}(t)|) d t \leqslant \int_{\mathbb{T}} \varphi_{d-1}^{0}(|\tilde{f}(t)|) d t+2 \pi \varphi_{d-1}(1) \\
\leqslant K \int_{\mathbb{T}}|f(u)| \int_{0}^{|f(u)|} t^{-2} \varphi_{d-1}^{0}(t) d t d u+K+2 \pi \varphi_{d-1}(1) \\
=K \int_{\{u \in \mathbb{T}:|f(u)|>1\}}|f(u)| \int_{1}^{|f(u)|} t^{-2} \varphi_{d-1}(t) d t d u \\
\quad+K+2 \pi \varphi_{d-1}(1) .
\end{aligned}
$$

Оценим внутренний интеграл в первом слагаемом правой части (2.21). Воспользовавшись неубыванием функции

$$
\frac{\varphi_{d-1}(u)}{u}=\psi(u)\left(\log \left(u+u_{d-1}\right)\right)^{d-2}
$$

для $u$ таких, что $|f(u)|>1$, получаем

$$
\begin{aligned}
\int_{1}^{|f(u)|} t^{-2} \varphi_{d-1}(t) d t & \leqslant \varphi_{d-1}(|f(u)|)|f(u)|^{-1}\left(\int_{1}^{|f(u)|} t^{-1} d t\right) \\
& =\psi(|f(u)|)\left(\log \left(|f(u)|+u_{d-1}\right)\right)^{d-2}\left(\int_{1}^{|f(u)|} t^{-1} d t\right) .
\end{aligned}
$$

Отсюда, учитывая, что

$$
\int_{1}^{|f(u)|} t^{-1} d t \leqslant \log \left(|f(u)|+u_{d-1}\right)
$$

и что $u_{d-1} \leqslant u_{d}$, заключаем:

$$
\begin{aligned}
\int_{1}^{|f(u)|} t^{-2} \varphi_{d-1}(t) d t & \leqslant \psi(|f(u)|)\left(\log \left(|f(u)|+u_{d}\right)\right)^{d-1} \\
& =|f(u)|^{-1} \varphi_{d}(|f(t)|) .
\end{aligned}
$$

Объединяя соотношения $(2.21)$ и $(2.22)$, получаем

$$
\begin{aligned}
\int_{\mathbb{T}} \varphi_{d-1}(|\tilde{f}(t)|) d t & \leqslant K \int_{\{u \in \mathbb{T}:|f(u)|>1\}} \varphi_{d}(|f(u)|) d u+K+2 \pi \varphi_{d-1}(1) \\
& \leqslant K_{d} \int_{\mathbb{T}} \varphi_{d}(|f(u)|) d u+K_{d}
\end{aligned}
$$

где $K_{d}=K+2 \pi \varphi_{d-1}(1)$. Лемма доказана. 
ЛЕмма 5. Пусть $g$ - кратный тригонометрический полином, определен-

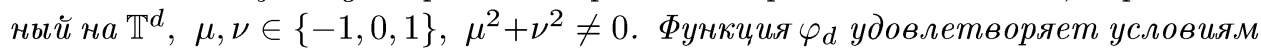
леммы 4. Тогда функиия $h=h^{\mu, \nu}$, определяемая равенством

$$
h^{\mu, \nu}\left(x_{1}, x_{2}, \ldots, x_{d}\right)=-\frac{1}{2 \pi} \int_{\mathbb{T}} g\left(x_{1}+\mu t, x_{2}+\nu t, x_{3}, \ldots, x_{d}\right) \operatorname{ctg}\left(\frac{t}{2}\right) d t
$$

где интеграл понимается в смысле главного значения, также является тригонометрическим полиномом и

$$
\begin{aligned}
\int_{\mathbb{T}^{d}} \varphi_{d-1}\left(\left|h\left(x_{1}, \ldots, x_{d}\right)\right|\right) d x_{1} \ldots d x_{d} \\
\quad \leqslant \bar{K}_{d} \int_{\mathbb{T}^{d}} \varphi_{d}\left(\left|g\left(x_{1}, \ldots, x_{d}\right)\right|\right) d x_{1} \ldots d x_{d}+\bar{K}_{d}
\end{aligned}
$$

Здесь $\bar{K}_{d}=\bar{K}_{d}(\psi)$ не зависит от $g$.

ДокАЗАТЕЛЬСтво. Тригонометрический полином $g$ представим в виде суммы слагаемых вида $c_{k} e^{i k x}, k=\left(k_{1}, \ldots, k_{d}\right) \in \mathbb{Z}^{d}, x=\left(x_{1}, \ldots, x_{d}\right) \in \mathbb{T}^{d}$. Нетрудно убедиться в том, что исследуемое преобразованиепереводит каждое из этих слагаемых в функцию того же вида, откуда следует, что $h$ является тригонометрическим полиномом.

Докажем теперь неравенство (2.23). Без ограничения общности можно считать, что $\mu \neq 0$. Введя новые переменные $x_{1}=x_{1}^{\prime}, x_{2}=x_{2}^{\prime}+(\nu / \mu) x_{1}^{\prime}, x_{3}=x_{3}^{\prime}, \ldots$ $\ldots, x_{d}=x_{d}^{\prime}$, имеем

$$
\begin{aligned}
& h\left(x_{1}, x_{2}, \ldots, x_{d}\right) \\
& \quad=-\frac{1}{2 \pi} \int_{\mathbb{T}} g\left(x_{1}^{\prime}+\mu t, x_{2}^{\prime}+\frac{\nu}{\mu} x_{1}^{\prime}+\nu t, x_{3}^{\prime}, \ldots, x_{d}^{\prime}\right) \operatorname{ctg}\left(\frac{t}{2}\right) d t \\
& \quad=-\frac{1}{2 \pi} \int_{\mathbb{T}} g\left(x_{1}^{\prime}+\mu t, x_{2}^{\prime}+\frac{\nu}{\mu}\left(x_{1}^{\prime}+\mu t\right), x_{3}^{\prime}, \ldots, x_{d}^{\prime}\right) \operatorname{ctg}\left(\frac{t}{2}\right) d t .
\end{aligned}
$$

При фиксированных $x_{2}^{\prime}, \ldots, x_{d}^{\prime}$ правая часть $(2.24)$ как функция переменной $x_{1}^{\prime}$ является (с точностью до знака) сопряженной к функции $g\left(x_{1}^{\prime}, x_{2}^{\prime}+(\nu / \mu) x_{1}^{\prime}\right.$, $\left.x_{3}^{\prime}, \ldots, x_{d}^{\prime}\right)$, поэтому из леммы 4 следует

$$
\begin{gathered}
\int_{\mathbb{T}} \varphi_{d-1}\left(\left|\frac{1}{2 \pi} \int_{\mathbb{T}} g\left(x_{1}^{\prime}+\mu t, x_{2}^{\prime}+\frac{\nu}{\mu}\left(x_{1}^{\prime}+\mu t\right), x_{3}^{\prime}, \ldots, x_{d}^{\prime}\right) \operatorname{ctg}\left(\frac{t}{2}\right) d t\right|\right) d x_{1}^{\prime} \\
\leqslant K_{d} \int_{\mathbb{T}} \varphi_{d}\left(\left|g\left(x_{1}^{\prime}, x_{2}^{\prime}+\frac{\nu}{\mu} x_{1}^{\prime}, x_{3}^{\prime}, \ldots, x_{d}^{\prime}\right)\right|\right) d x_{1}^{\prime}+K_{d} \\
\left(x_{2}^{\prime}, \ldots, x_{d}^{\prime}\right) \in \mathbb{T}^{d-1} .
\end{gathered}
$$

Интегрируя $(2.25)$ по $\left(x_{2}^{\prime}, \ldots, x_{d}^{\prime}\right) \in \mathbb{T}^{d-1}$, пользуясь равенством $(2.24)$ и замечая, что якобиан перехода от $\left(x_{1}^{\prime}, x_{2}^{\prime}, \ldots, x_{d}^{\prime}\right)$ к $\left(x_{1}, x_{2}, \ldots, x_{d}\right)$ равен единище, получаем (2.23). Лемма доказана. 


\section{§3. Доказательство теоремы 1}

Напомним, что через $M_{B}(f, x)$ мы обозначаем определенную равенством $(2.18)$ максимальную функцию частичных сумм $B$-сопряженного ряда Фурье функции $f$.

Докажем индукцией по $d$, что при условиях теоремы 1 найдутся константы $A_{d}>0$ и $y_{0}>0$ такие, что для любого кратного тригонометрического полинома $g$, определенного на $\mathbb{T}^{d}$, для всех $B \subset\{1, \ldots, d\}$ и любых $y \geqslant y_{0}$ справедливо неравенство

$$
m\left\{x \in \mathbb{T}^{d}: M_{B}(g, x)>y\right\} \leqslant \frac{A_{d}}{y}\left(\int_{\mathbb{T}^{d}} \varphi_{d}(|g(t)|) d t+1\right)
$$

Утверждение теоремы тогда будет вытекать из (3.1) в силу леммы 3.

База индукиии. Согласно условию теоремы ряд Фурье любой функции $f \in$ $\varphi_{1}(L)(\mathbb{T})$ сходится почти всюду. Следовательно (см., например, [14, с. 262-263, теорема 5.1]), ряд, сопряженный к ряду Фурье функции $f$, также сходится почти всюду. Применяя лемму 3 при $d=1, B=\{\varnothing\}$ и $d=1, B=\{1\}$, получаем (3.1) для $d=1$.

Шаг индукиии. Пусть $d \geqslant 2$. Предположим, что (3.1) верно для всех размерностей, меньших $d$. Пусть $g(t)=g\left(t_{1}, t_{2}, \ldots, t_{d}\right)$ - произвольный тригонометрический полином и $B$ - некоторое подмножество множества $N_{d}=\{1,2, \ldots, d\}$. Обозначим $\bar{B}=N_{d} \backslash B$. Рассмотрим три случая.

Случай 1 . Пусть $1 \in \bar{B}, 2 \in \bar{B}$. Обозначим

$$
\Pi_{n}=\Pi_{n}\left(t_{3}, \ldots, t_{d}\right)=\prod_{i \in B}\left(-\widetilde{D}_{n}\left(t_{i}\right)\right) \prod_{j \in \bar{B} \backslash\{1,2\}} D_{n}\left(t_{j}\right) .
$$

Тогда

$$
\widetilde{S}_{n, B}(g, x)=\frac{1}{\pi^{d}} \int_{\mathbb{T}^{d}} D_{n}\left(t_{1}\right) D_{n}\left(t_{2}\right) \Pi_{n}\left(t_{3}, \ldots, t_{d}\right) g(x+t) d t .
$$

Применяя последовательно тригонометрические тождества

$$
\sin \left(\alpha t_{1}\right) \sin \left(\alpha t_{2}\right)=\frac{1}{2}\left[\cos \alpha\left(t_{1}-t_{2}\right)-\cos \alpha\left(t_{1}+t_{2}\right)\right]
$$

при $\alpha=1 / 2$ и $\alpha=n+1 / 2$ и

$$
\frac{1}{2 \sin \left(\frac{t_{1}}{2}\right) \cdot 2 \sin \left(\frac{t_{2}}{2}\right)}=\frac{1}{2 \sin \left(\frac{t_{1} \pm t_{2}}{2}\right)}\left(\frac{1}{2} \operatorname{ctg}\left(\frac{t_{2}}{2}\right) \pm \frac{1}{2} \operatorname{ctg}\left(\frac{t_{1}}{2}\right)\right)
$$

при $t_{1}, t_{2}$ таких, что $t_{1} \pm t_{2} \neq 2 \pi k, k \in \mathbb{Z}$, получаем

$$
\begin{aligned}
& D_{n}\left(t_{1}\right) D_{n}\left(t_{2}\right)=\frac{\sin \left(n+\frac{1}{2}\right) t_{1} \cdot \sin \left(n+\frac{1}{2}\right) t_{2}}{2 \sin \left(\frac{t_{1}}{2}\right) \cdot 2 \sin \left(\frac{t_{2}}{2}\right)} \\
&+\frac{\frac{1}{2} \cos \left(\frac{t_{1}+t_{2}}{2}\right)-\frac{1}{2} \cos \left(\frac{t_{1}-t_{2}}{2}\right)+\sin \left(\frac{t_{1}}{2}\right) \sin \left(\frac{t_{2}}{2}\right)}{2 \sin \left(\frac{t_{1}}{2}\right) \cdot 2 \sin \left(\frac{t_{2}}{2}\right)}
\end{aligned}
$$




$$
\begin{aligned}
= & \frac{1}{2} \frac{\cos \left(n+\frac{1}{2}\right)\left(t_{1}-t_{2}\right)-\cos \left(\frac{t_{1}-t_{2}}{2}\right)}{2 \sin \left(\frac{t_{1}}{2}\right) \cdot 2 \sin \left(\frac{t_{2}}{2}\right)} \\
& -\frac{1}{2} \frac{\cos \left(n+\frac{1}{2}\right)\left(t_{1}+t_{2}\right)-\cos \left(\frac{t_{1}+t_{2}}{2}\right)}{2 \sin \left(\frac{t_{1}}{2}\right) \cdot 2 \sin \left(\frac{t_{2}}{2}\right)}+\frac{1}{4} \\
= & \frac{1}{2} \frac{\cos \left(n+\frac{1}{2}\right)\left(t_{1}-t_{2}\right)-\cos \left(\frac{t_{1}-t_{2}}{2}\right)}{2 \sin \left(\frac{t_{1}-t_{2}}{2}\right)}\left(\frac{1}{2} \operatorname{ctg}\left(\frac{t_{2}}{2}\right)-\frac{1}{2} \operatorname{ctg}\left(\frac{t_{1}}{2}\right)\right) \\
& -\frac{1}{2} \frac{\cos \left(n+\frac{1}{2}\right)\left(t_{1}+t_{2}\right)-\cos \left(\frac{t_{1}+t_{2}}{2}\right)}{2 \sin \left(\frac{t_{1}+t_{2}}{2}\right)}\left(\frac{1}{2} \operatorname{ctg}\left(\frac{t_{2}}{2}\right)+\frac{1}{2} \operatorname{ctg}\left(\frac{t_{1}}{2}\right)\right)+\frac{1}{4} \\
= & \frac{1}{2}\left[\frac{\cos \left(n+\frac{1}{2}\right)\left(t_{1}-t_{2}\right)-\cos \left(\frac{t_{1}-t_{2}}{2}\right)}{2 \sin \left(\frac{t_{1}-t_{2}}{2}\right)}\right. \\
& \left.-\frac{\cos \left(n+\frac{1}{2}\right)\left(t_{1}+t_{2}\right)-\cos \left(\frac{t_{1}+t_{2}}{2}\right)}{2 \sin \left(\frac{t_{1}+t_{2}}{2}\right)}\right] \cdot \frac{1}{2} \operatorname{ctg}\left(\frac{t_{2}}{2}\right) \\
& +\frac{1}{2}\left[\frac{\cos \left(n+\frac{1}{2}\right)\left(t_{2}-t_{1}\right)-\cos \left(\frac{t_{2}-t_{1}}{2}\right)}{2 \sin \left(\frac{t_{2}-t_{1}}{2}\right)}\right. \\
& \left.-\frac{\cos \left(n+\frac{1}{2}\right)\left(t_{2}+t_{1}\right)-\cos \left(\frac{t_{2}+t_{1}}{2}\right)}{2 \sin \left(\frac{t_{2}+t_{1}}{2}\right)}\right] \cdot \frac{1}{2} \operatorname{ctg}\left(\frac{t_{1}}{2}\right)+\frac{1}{4} .
\end{aligned}
$$

Таким образом,

$$
D_{n}\left(t_{1}\right) D_{n}\left(t_{2}\right)=D_{n}^{1}+D_{n}^{2}+\frac{1}{4}
$$

где

$$
\begin{aligned}
& D_{n}^{1}=\frac{1}{2}\left[\widetilde{D}_{n}\left(t_{2}+t_{1}\right)-\widetilde{D}_{n}\left(t_{2}-t_{1}\right)\right] \cdot \frac{1}{2} \operatorname{ctg}\left(\frac{t_{1}}{2}\right), \\
& D_{n}^{2}=\frac{1}{2}\left[\widetilde{D}_{n}\left(t_{1}+t_{2}\right)-\widetilde{D}_{n}\left(t_{1}-t_{2}\right)\right] \cdot \frac{1}{2} \operatorname{ctg}\left(\frac{t_{2}}{2}\right) .
\end{aligned}
$$

Заметим, что при каждом $n$ функции $D_{n}^{1}$ и $D_{n}^{2}$ ограничены при $\left(t_{1}, t_{2}\right) \in \mathbb{T}^{2}$.

Обе части равенства (3.4) умножаем на $\Pi_{n} g\left(x_{1}+t_{1}, \ldots, x_{d}+t_{d}\right)$ и интегрируем по множеству $\left\{\left(t_{1}, t_{2}, \ldots, t_{d}\right) \in \mathbb{T}^{d}:\left|t_{1}\right|>\varepsilon\right\}$ при произвольном $\left(x_{1}, x_{2}, \ldots, x_{d}\right) \in \mathbb{T}^{d}$. Затем разобьем получившийся в правой части интеграл на разность двух интегралов в соответствии с разностью в (3.4), сделаем в каждом из них замену переменных соответственно $u_{1}=t_{1}, u_{2}=t_{2}+t_{1}, u_{3}=t_{3}, \ldots$ $\ldots, u_{d}=t_{d}$ и $u_{1}=t_{1}, u_{2}=t_{2}-t_{1}, u_{3}=t_{3}, \ldots, u_{d}=t_{d}$ и перейдем к пределу при $\varepsilon \rightarrow 0$. Получим равенство

$$
\int_{\mathbb{T}^{d}} D_{n}^{1} \Pi_{n} g\left(x_{1}+t_{1}, \ldots, x_{d}+t_{d}\right) d t=\frac{1}{2} \int_{\mathbb{T}^{d-1}} \widetilde{D}_{n}\left(u_{2}\right) \Pi_{n}\left(u_{3}, \ldots, u_{d}\right)
$$




$$
\begin{aligned}
& \times\left(\int_{\mathbb{T}} g\left(x_{1}+u_{1}, x_{2}+u_{2}-u_{1}, x_{3}+u_{3}, \ldots, x_{d}+u_{d}\right)\right. \\
& \left.\times \frac{1}{2} \operatorname{ctg}\left(\frac{u_{1}}{2}\right) d u_{1}\right) d u_{2} \ldots d u_{d}-\frac{1}{2} \int_{\mathbb{T}^{d-1}} \widetilde{D}_{n}\left(u_{2}\right) \Pi_{n}\left(u_{3}, \ldots, u_{d}\right) \\
& \times\left(\int_{\mathbb{T}} g\left(x_{1}+u_{1}, x_{2}+u_{2}+u_{1}, x_{3}+u_{3}, \ldots, x_{d}+u_{d}\right)\right. \\
& \left.\times \frac{1}{2} \operatorname{ctg}\left(\frac{u_{1}}{2}\right) d u_{1}\right) d u_{2} \ldots d u_{d} .
\end{aligned}
$$

Внутренние интегралы в правой части (3.6), понимаемые в смысле главного значения, согласно обозначениям леммы 5 можно записать как $-\pi h^{1,-1}\left(x_{1}, x_{2}+u_{2}\right.$, $\left.x_{3}+u_{3}, \ldots, x_{d}+u_{d}\right)$ и $-\pi h^{1,1}\left(x_{1}, x_{2}+u_{2}, x_{3}+u_{3}, \ldots, x_{d}+u_{d}\right)$. Проделав с равенством (3.5) то же, что и с (3.4), только производя при этом замены $u_{1}=$ $t_{1}+t_{2}, u_{2}=t_{2}, \ldots, u_{d}=t_{d}$ и $u_{1}=t_{1}-t_{2}, u_{2}=t_{2}, \ldots, u_{d}=t_{d}$, получаем

$$
\begin{aligned}
\int_{\mathbb{T}^{d}} D_{n}^{2} & \Pi_{n} g\left(x_{1}+t_{1}, \ldots, x_{d}+t_{d}\right) d t \\
= & \frac{1}{2} \int_{\mathbb{T}^{d-1}} \widetilde{D}_{n}\left(u_{1}\right) \Pi_{n}\left(u_{3}, \ldots, u_{d}\right) \\
& \times\left(\int_{\mathbb{T}} g\left(x_{1}+u_{1}-u_{2}, x_{2}+u_{2}, x_{3}+u_{3}, \ldots, x_{d}+u_{d}\right)\right. \\
& \left.\times \frac{1}{2} \operatorname{ctg}\left(\frac{u_{2}}{2}\right) d u_{2}\right) d u_{1} d u_{3} \ldots d u_{d}-\frac{1}{2} \int_{\mathbb{T}^{d-1}} \widetilde{D}_{n}\left(u_{1}\right) \Pi_{n}\left(u_{3}, \ldots, u_{d}\right) \\
& \times\left(\int_{\mathbb{T}} g\left(x_{1}+u_{1}+u_{2}, x_{2}+u_{2}, x_{3}+u_{3}, \ldots, x_{d}+u_{d}\right)\right. \\
& \left.\times \frac{1}{2} \operatorname{ctg}\left(\frac{u_{2}}{2}\right) d u_{2}\right) d u_{1} d u_{3} \ldots d u_{d} \\
= & -\frac{\pi}{2} \int_{\mathbb{T}^{d-1}} \widetilde{D}_{n}\left(u_{1}\right) \Pi_{n}\left(u_{3}, \ldots, u_{d}\right) \\
& \times h^{-1,1}\left(x_{1}+u_{1}, x_{2}, x_{3}+u_{3}, \ldots, x_{d}+u_{d}\right) d u_{1} d u_{3} \ldots d u_{d} \\
& +\frac{\pi}{2} \int_{\mathbb{T}^{d-1}} \widetilde{D}_{n}\left(u_{1}\right) \Pi_{n}\left(u_{3}, \ldots, u_{d}\right) \\
& \times h^{1,1}\left(x_{1}+u_{1}, x_{2}, x_{3}+u_{3}, \ldots, x_{d}+u_{d}\right) d u_{1} d u_{3} \ldots d u_{d}
\end{aligned}
$$

Используя определение $M_{B}(g, x)$, соотношения (3.2), (3.3), (3.6) и (3.7), имеем

$$
\begin{aligned}
2 M_{B}(g, x) \leqslant & \max _{n \in \mathbb{N}} \mid \frac{1}{\pi^{d-1}} \int_{\mathbb{T}^{d-1}} \widetilde{D}_{n}\left(u_{2}\right) \Pi_{n}\left(u_{3}, \ldots, u_{d}\right) \\
& \times h^{1,-1}\left(x_{1}, x_{2}+u_{2}, x_{3}+u_{3}, \ldots, x_{d}+u_{d}\right) d u_{2} \ldots d u_{d} \mid \\
& +\max _{n \in \mathbb{N}} \mid \frac{1}{\pi^{d-1}} \int_{\mathbb{T}^{d-1}} \widetilde{D}_{n}\left(u_{2}\right) \Pi_{n}\left(u_{3}, \ldots, u_{d}\right) \\
& \times h^{1,1}\left(x_{1}, x_{2}+u_{2}, x_{3}+u_{3}, \ldots, x_{d}+u_{d}\right) d u_{2} \ldots d u_{d} \mid
\end{aligned}
$$




$$
\begin{aligned}
& +\max _{n \in \mathbb{N}} \mid \frac{1}{\pi^{d-1}} \int_{\mathbb{T}^{d-1}} \widetilde{D}_{n}\left(u_{1}\right) \Pi_{n}\left(u_{3}, \ldots, u_{d}\right) \\
& \times h^{-1,1}\left(x_{1}+u_{1}, x_{2}, x_{3}+u_{3}, \ldots, x_{d}+u_{d}\right) d u_{1} d u_{3} \ldots d u_{d} \mid \\
& +\max _{n \in \mathbb{N}} \mid \frac{1}{\pi^{d-1}} \int_{\mathbb{T}^{d-1}} \widetilde{D}_{n}\left(u_{1}\right) \Pi_{n}\left(u_{3}, \ldots, u_{d}\right) \\
& \quad \times h^{1,1}\left(x_{1}+u_{1}, x_{2}, x_{3}+u_{3}, \ldots, x_{d}+u_{d}\right) d u_{1} d u_{3} \ldots d u_{d} \mid \\
& +\max _{n \in \mathbb{N}}\left|\frac{1}{2 \pi^{d}} \int_{\mathbb{T}^{d}} \Pi_{n}\left(u_{3}, \ldots, u_{d}\right) g\left(x_{1}+t_{1}, \ldots, x_{d}+t_{d}\right) d t_{1} \ldots d t_{d}\right| \\
& =M_{B}^{1}(g, x)+M_{B}^{2}(g, x)+M_{B}^{3}(g, x)+M_{B}^{4}(g, x)+M_{B}^{5}(g, x) .
\end{aligned}
$$

Покажем, что $M_{B}^{1}(g, x)$ удовлетворяет неравенству (3.1) с некоторой константой $A_{d}^{1}$.

Зафиксируем $x_{1} \in \mathbb{T}$. Согласно предположению индукции для полинома $h^{1,-1}\left(x_{1}, x_{2}, \ldots, x_{d}\right)$ как функции $d-1$ переменных $x_{2}, \ldots, x_{d}$ и для $B^{\prime}=$ $B \backslash\{1\} \subset\{2,3, \ldots, d\}$ справедлива оценка

$$
\begin{gathered}
m\left\{\left(x_{2}, \ldots, x_{d}\right) \in \mathbb{T}^{d-1}: \max _{n \in \mathbb{N}} \mid \frac{1}{\pi^{d-1}} \int_{\mathbb{T}^{d-1}} \widetilde{D}_{n}\left(u_{2}\right) \Pi_{n}\left(u_{3}, \ldots, u_{d}\right)\right. \\
\left.\times h^{1,-1}\left(x_{1}, x_{2}+u_{2}, x_{3}+u_{3}, \ldots, x_{d}+u_{d}\right) d u_{2} \ldots d u_{d} \mid>y\right\} \\
=m\left\{\left(x_{2}, \ldots, x_{d}\right) \in \mathbb{T}^{d-1}: \max _{n \in \mathbb{N}}\left|S_{n, B^{\prime}}\left(h^{1,-1}\left(x_{2}, \ldots, x_{d}\right)\right)\right|>y\right\} \\
\leqslant \frac{A_{d-1}}{y}\left(\int_{\mathbb{T}^{d-1}} \varphi_{d-1}\left(\left|h^{1,-1}\left(x_{1}, x_{2}, x_{3}, \ldots, x_{d}\right)\right|\right) d x_{2} \ldots d x_{d}+1\right), \\
y>y_{0} .
\end{gathered}
$$

Пользуясь тем, что

$$
\begin{aligned}
& m\left\{\left(x_{1}, x_{2}, \ldots, x_{d}\right) \in \mathbb{T}^{d}: M_{B}^{1}(g, x)>y\right\} \\
& =\int_{\mathbb{T}} m\left\{\left(x_{2}, \ldots, x_{d}\right) \in \mathbb{T}^{d-1}: \max _{n \in \mathbb{N}} \mid \frac{1}{\pi^{d-1}} \int_{\mathbb{T}^{d-1}} \widetilde{D}_{n}\left(u_{2}\right) \Pi_{n}\left(u_{3}, \ldots, u_{d}\right)\right. \\
& \left.\quad \times h^{1,-1}\left(x_{1}, x_{2}+u_{2}, x_{3}+u_{3}, \ldots, x_{d}+u_{d}\right) d u_{2} \ldots d u_{d} \mid>y\right\} d x_{1},
\end{aligned}
$$

применяя (3.9), а затем теорему Фубини, получаем

$$
\begin{gathered}
m\left\{\left(x_{1}, x_{2}, \ldots, x_{d}\right) \in \mathbb{T}^{d}: M_{B}^{1}(g, x)>y\right\} \\
\leqslant \frac{A_{d-1}}{y} \int_{\mathbb{T}}\left(\int_{\mathbb{T}^{d-1}} \varphi_{d-1}\left(\left|h^{1,-1}\left(x_{1}, x_{2}, x_{3}, \ldots, x_{d}\right)\right|\right) d x_{2} \ldots d x_{d}+1\right) d x_{1} \\
=\frac{A_{d-1}}{y}\left(\int_{\mathbb{T}^{d}} \varphi_{d-1}\left(\left|h^{1,-1}\left(x_{1}, x_{2}, x_{3}, \ldots, x_{d}\right)\right|\right) d x_{1} d x_{2} \ldots d x_{d}+2 \pi\right), \\
y>y_{0} .
\end{gathered}
$$


Отсюда и из леммы 5 заключаем

$$
\begin{aligned}
& m\left\{\left(x_{1}, x_{2}, \ldots, x_{d}\right) \in \mathbb{T}^{d}: M_{B}^{1}(g, x)>y\right\} \\
& \quad \leqslant \frac{A_{d-1}}{y}\left(\bar{K}_{d} \int_{\mathbb{T}^{d}} \varphi_{d}\left(\left|g\left(x_{1}, x_{2}, \ldots, x_{d}\right)\right|\right) d x_{1} d x_{2} \ldots d x_{d}+\bar{K}_{d}+2 \pi\right) \\
& \quad \leqslant \frac{A_{d}^{1}}{y}\left(\int_{\mathbb{T}^{d}} \varphi_{d}\left(\left|g\left(x_{1}, x_{2}, \ldots, x_{d}\right)\right|\right) d x_{1} d x_{2} \ldots d x_{d}+1\right), \quad y>y_{0},
\end{aligned}
$$

где $A_{d}^{1}=A_{d-1}\left(\bar{K}_{d}+2 \pi\right)$.

Аналогично можно показать, что $M_{B}^{i}(g, x), i=2,3,4$, также удовлетворяют (3.1) с той же константой $A_{d}^{1}$.

Рассмотрим слагаемое $M_{B}^{5}(g, x)$. При $d=2$ имеем $\Pi_{n} \equiv 1$, поэтому

$$
M_{B}^{5}(g, x) \leqslant \frac{1}{2 \pi^{d}} \int_{\mathbb{T}^{d}}|g(t)| d t
$$

откуда в силу неубывания $\varphi_{2}(u) / u$ следует неравенство $(3.1)$ с некоторой константой $A_{2}^{5}=A_{2}^{5}(\psi)$. Если $d>2$, то неравенство $(3.1)$ следует из равенства

$$
\begin{aligned}
m & \left\{\left(x_{1}, x_{2}, \ldots, x_{d}\right) \in \mathbb{T}^{d}: M_{B}^{5}(g, x)>y\right\} \\
= & \int_{\mathbb{T}^{2}} m\left\{\left(x_{3}, \ldots, x_{d}\right) \in \mathbb{T}^{d-2}: \max _{n \in \mathbb{N}} \mid \frac{1}{2 \pi^{d}} \int_{\mathbb{T}^{d-2}} \Pi_{n}\left(u_{3}, \ldots, u_{d}\right)\right. \\
& \left.\times\left(\int_{\mathbb{T}^{2}} g\left(x_{1}+t_{1}, x_{2}+t_{2}, x_{3}+t_{3}, \ldots, x_{d}+t_{d}\right) d t_{1} d t_{2}\right) d t_{3} \ldots d t_{d} \mid>y\right\} d x_{1} d x_{2}
\end{aligned}
$$

и предположения индукции, примененного для размерности $d-2$ к полиному

$$
h_{x_{1}, x_{2}}\left(t_{3}, \ldots, t_{d}\right)=\frac{1}{2 \pi^{2}} \int_{\mathbb{T}^{2}} g\left(x_{1}+t_{1}, x_{2}+t_{2}, t_{3}, \ldots, t_{d}\right) d t_{1} d t_{2}
$$

Таким образом, для $M_{B}(g, x)$ неравенство (3.1) выполняется при $A_{d}=$ $4 A_{d}^{1}+A_{d}^{5}$.

Случай 2. Пусть $1 \in B, 2 \in \bar{B}$. (Случай, когда $1 \in \bar{B}, 2 \in B$, рассматривается аналогично.) Здесь

$$
\widetilde{S}_{n, B}(g, x)=-\frac{1}{\pi^{d}} \int_{\mathbb{T}^{d}} \widetilde{D}_{n}\left(t_{1}\right) D_{n}\left(t_{2}\right) \Pi_{n}\left(t_{3}, \ldots, t_{d}\right) g(x+t) d t
$$

Применяя последовательно тригонометрические тождества аналогично предыдущему случаю, получаем

$$
\begin{aligned}
& \frac{\cos \left(n+\frac{1}{2}\right) t_{1}-\cos \left(\frac{t_{1}}{2}\right)}{2 \sin \left(\frac{t_{1}}{2}\right)} \frac{\sin \left(n+\frac{1}{2}\right) t_{2}}{2 \sin \left(\frac{t_{2}}{2}\right)} \\
& \quad=\frac{\frac{1}{2} \sin \left(n+\frac{1}{2}\right)\left(t_{2}-t_{1}\right)+\frac{1}{2} \sin \left(n+\frac{1}{2}\right)\left(t_{2}+t_{1}\right)}{2 \sin \left(\frac{t_{1}}{2}\right) \cdot 2 \sin \left(\frac{t_{2}}{2}\right)}-\frac{\sin \left(n+\frac{1}{2}\right) t_{2}}{2 \sin \left(\frac{t_{2}}{2}\right)} \cdot \frac{1}{2} \operatorname{ctg}\left(\frac{t_{1}}{2}\right)
\end{aligned}
$$




$$
\begin{aligned}
= & \frac{1}{2} \frac{\sin \left(n+\frac{1}{2}\right)\left(t_{2}-t_{1}\right)}{2 \sin \left(\frac{t_{1}-t_{2}}{2}\right)}\left(\frac{1}{2} \operatorname{ctg}\left(\frac{t_{2}}{2}\right)-\frac{1}{2} \operatorname{ctg}\left(\frac{t_{1}}{2}\right)\right) \\
& +\frac{1}{2} \frac{\sin \left(n+\frac{1}{2}\right)\left(t_{2}+t_{1}\right)}{2 \sin \left(\frac{t_{2}+t_{1}}{2}\right)}\left(\frac{1}{2} \operatorname{ctg}\left(\frac{t_{2}}{2}\right)+\frac{1}{2} \operatorname{ctg}\left(\frac{t_{1}}{2}\right)\right) \\
& -\frac{\sin \left(n+\frac{1}{2}\right) t_{2}}{2 \sin \left(\frac{t_{2}}{2}\right)} \cdot \frac{1}{2} \operatorname{ctg}\left(\frac{t_{1}}{2}\right) \\
= & \frac{1}{2}\left[\frac{\sin \left(n+\frac{1}{2}\right)\left(t_{2}+t_{1}\right)}{2 \sin \left(\frac{t_{2}+t_{1}}{2}\right)}+\frac{\sin \left(n+\frac{1}{2}\right)\left(t_{2}-t_{1}\right)}{2 \sin \left(\frac{t_{2}-t_{1}}{2}\right)}\right. \\
& \left.-\frac{\sin \left(n+\frac{1}{2}\right) t_{2}}{\sin \left(\frac{t_{2}}{2}\right)}\right] \cdot \frac{1}{2} \operatorname{ctg}\left(\frac{t_{1}}{2}\right) \\
& +\frac{1}{2}\left[\frac{\sin \left(n+\frac{1}{2}\right)\left(t_{1}+t_{2}\right)}{2 \sin \left(\frac{t_{1}+t_{2}}{2}\right)}-\frac{\sin \left(n+\frac{1}{2}\right)\left(t_{1}-t_{2}\right)}{2 \sin \left(\frac{t_{1}-t_{2}}{2}\right)}\right] \cdot \frac{1}{2} \operatorname{ctg}\left(\frac{t_{2}}{2}\right),
\end{aligned}
$$

т. e.

$$
\widetilde{D}_{n}\left(t_{1}\right) D_{n}\left(t_{2}\right)=D_{n}^{3}+D_{n}^{4}
$$

где

$$
\begin{gathered}
D_{n}^{3}=\frac{1}{2}\left[D_{n}\left(t_{2}+t_{1}\right)+D_{n}\left(t_{2}-t_{1}\right)-2 D_{n}\left(t_{2}\right)\right] \cdot \frac{1}{2} \operatorname{ctg}\left(\frac{t_{1}}{2}\right), \\
D_{n}^{4}=\frac{1}{2}\left[D_{n}\left(t_{1}+t_{2}\right)-D_{n}\left(t_{1}-t_{2}\right)\right] \cdot \frac{1}{2} \operatorname{ctg}\left(\frac{t_{2}}{2}\right) .
\end{gathered}
$$

Теперь обе части равенства (3.12) умножаем на $\Pi_{n} g\left(x_{1}+t_{1}, \ldots, x_{d}+t_{d}\right)$ и интегрируем по множеству $\left\{\left(t_{1}, t_{2}, \ldots, t_{d}\right) \in \mathbb{T}^{d}:\left|t_{1}\right|>\varepsilon\right\}$ при произвольном $\left(x_{1}, x_{2}, \ldots, x_{d}\right) \in \mathbb{T}^{d}$. Затем разобьем получившийся в правой части интеграл на три интеграла, сделаем в первых двух из них замену переменных соответственно $u_{1}=t_{1}, u_{2}=t_{2}+t_{1}, u_{3}=t_{3}, \ldots, u_{d}=t_{d}$ и $u_{1}=t_{1}, u_{2}=t_{2}-t_{1}, u_{3}=t_{3}, \ldots$ $\ldots, u_{d}=t_{d}$ и перейдем к пределу при $\varepsilon \rightarrow 0$. Аналогичные действия произведем с равенством (3.13). Используя получившиеся соотношения, а также (3.11) и $(3.10)$, имеем

$$
\begin{aligned}
2 M_{B}(g, x) \leqslant & \max _{n \in \mathbb{N}} \mid \frac{1}{\pi^{d-1}} \int_{\mathbb{T}^{d-1}} \widetilde{D}_{n}\left(u_{2}\right) \Pi_{n}\left(u_{3}, \ldots, u_{d}\right) \\
& \times h^{1,-1}\left(x_{1}, x_{2}+u_{2}, x_{3}+u_{3}, \ldots, x_{d}+u_{d}\right) d u_{2} \ldots d u_{d} \mid \\
& +\max _{n \in \mathbb{N}} \mid \frac{1}{\pi^{d-1}} \int_{\mathbb{T}^{d-1}} \widetilde{D}_{n}\left(u_{2}\right) \Pi_{n}\left(u_{3}, \ldots, u_{d}\right) \\
& \times h^{1,1}\left(x_{1}, x_{2}+u_{2}, x_{3}+u_{3}, \ldots, x_{d}+u_{d}\right) d u_{2} \ldots d u_{d} \mid \\
& +\max _{n \in \mathbb{N}} \mid \frac{1}{\pi^{d-1}} \int_{\mathbb{T}^{d-1}} \widetilde{D}_{n}\left(u_{2}\right) \Pi_{n}\left(u_{3}, \ldots, u_{d}\right) \\
& \times h^{1,0}\left(x_{1}, x_{2}+u_{2}, x_{3}+u_{3}, \ldots, x_{d}+u_{d}\right) d u_{2} \ldots d u_{d} \mid
\end{aligned}
$$




$$
\begin{aligned}
& +\max _{n \in \mathbb{N}} \mid \frac{1}{\pi^{d-1}} \int_{\mathbb{T}^{d-1}} \widetilde{D}_{n}\left(u_{1}\right) \Pi_{n}\left(u_{3}, \ldots, u_{d}\right) \\
& \times h^{-1,1}\left(x_{1}+u_{1}, x_{2}, x_{3}+u_{3}, \ldots, x_{d}+u_{d}\right) d u_{1} d u_{3} \ldots d u_{d} \mid \\
& +\max _{n \in \mathbb{N}} \mid \frac{1}{\pi^{d-1}} \int_{\mathbb{T}^{d-1}} \widetilde{D}_{n}\left(u_{1}\right) \Pi_{n}\left(u_{3}, \ldots, u_{d}\right) \\
& \times h^{1,1}\left(x_{1}+u_{1}, x_{2}, x_{3}+u_{3}, \ldots, x_{d}+u_{d}\right) d u_{1} d u_{3} \ldots d u_{d} \mid .
\end{aligned}
$$

Используя те же рассуждения, что и в случае 1 , можно показать, что все пять слагаемых в правой части (3.14) удовлетворяют (3.1), откуда следует (3.1) для $M_{B}(g, x)$.

Случай 3 . Пусть $1 \in B, 2 \in B$. Этот случай рассматривается аналогично двум предыдушим. Здесь используется тождество

$$
\widetilde{D}_{n}\left(t_{1}\right) \widetilde{D}_{n}\left(t_{2}\right)=D_{n}^{5}+D_{n}^{6},
$$

где

$$
\begin{aligned}
& D_{n}^{5}=\frac{1}{2}\left[\widetilde{D}_{n}\left(t_{2}+t_{1}\right)+\widetilde{D}_{n}\left(t_{2}-t_{1}\right)-2 \widetilde{D}_{n}\left(t_{2}\right)\right] \cdot \frac{1}{2} \operatorname{ctg}\left(\frac{t_{1}}{2}\right), \\
& D_{n}^{6}=\frac{1}{2}\left[\widetilde{D}_{n}\left(t_{1}+t_{2}\right)+\widetilde{D}_{n}\left(t_{1}-t_{2}\right)-2 \widetilde{D}_{n}\left(t_{1}\right)\right] \cdot \frac{1}{2} \operatorname{ctg}\left(\frac{t_{2}}{2}\right) .
\end{aligned}
$$

Таким образом, (3.1) справедливо для любого $d \in \mathbb{N}$, всех кратных тригонометрических полиномов, определенных на $\mathbb{T}^{d}$, и произвольного $B \subset\{1,2, \ldots, d\}$. Теорема доказана.

Результаты этой работы были ранее депонированы в [15].

\section{Список литературы}

1. Carleson L. On convergence and growth of partial sums of Fourier series // Acta Math. 1966. V. 116. № 1-2. P. 135-157.

2. Hunt R.A. On the convergence of Fourier series // Orthogonal expansions and their continuous analogues. Carbondale-Illinois: SIU Press, 1968. P. 235-255.

3. Sjölin P. An inequality of Paley and convergence a.e. of Walsh-Fourier series // Arkiv för mat. 1969. V. 7. P. 551-570.

4. Antonov N. Yu. Convergence of Fourier series // East J. on Approx. 1996. V. 2. № 2. P. $187-196$.

5. Конягин С. В. О расходимости всюду тригонометрических рядов Фурье // Матем. сб. 2000. T. 191. № 1. C. 103-126.

6. Тевзадзе Н. Р. О сходимости двойного ряда Фурье функции, суммируемой с квадратом // Сообщ. АН ГССР. 1970. Т. 58. № 2. С. 277-279.

7. Fefferman C. On the convergence of multiple Fourier series // Bull. Amer. Math. Soc. 1971. V. 77. № 5. P. 744-745.

8. Sjölin P. Convergence almost everywhere of sertain singular integrals and multiple Fourier series // Arkiv för mat. 1971. V. 9. № 1. P. 65-90.

9. Konyagin S. V. On divergence of trigonometric Fourier series over cubes // Acta Sci. Math. (Szeged). 1995. V. 61. P. 305-329. 
10. Ульянов П. Л. Представление функций рядами и классы $\varphi(L) / /$ УМН. 1972. Т. 27. № 2. C. $3-52$.

11. Stein E. M. On limits of sequences of operators // Annals of Math. 1961. V. 74. № 1 . P. $140-170$.

12. Kolmogoroff $A$. Sur les fonctions harmoniques conjuguées et les séries de Fourier // Fund. Math. 1925. V. 7. P. 24-29.

13. Жижсиашвили Л. В. О справедливости теоремы А.Н. Колмогорова для сопряженных функций многих переменных // Матем. заметки. 1982. Т. 32. № 1. С. 13-21.

14. Зигмунд А. Тригонометрические ряды. Т. 2. М.: Мир, 1965.

15. Антонов Н.Ю. Сходимость почти всюду кратных рядов Фурье. Деп. в ВИНИТИ 24.11.97, № 3444-B97.

E-mail: Nikolai.Antonov@imm.uran.ru

Поступило в редакцию

24.11.2002 